\title{
Ginsenoside Rd as a potential neuroprotective agent prevents trimethyltin injury
}

\author{
JINGANG HOU $^{1 *}$, JIANJIE XUE $^{2 *}$, MIRA LEE $^{3}$ and CHANGKEUN SUNG ${ }^{4}$ \\ ${ }^{1}$ Intelligent Synthetic Biology Center, Daejeon 34141, Republic of Korea; \\ ${ }^{2}$ Qingdao Jingcheng Detection Technology Co., Ltd., Qingdao, Shandong 266555, P.R. China; \\ ${ }^{3}$ Korea Institute of Oriental Medicine, Daegu $41062 ;{ }^{4}$ Department of Food Science and Technology, \\ College of Agriculture and Biotechnology, Chungnam National University, Daejeon 305764, Republic of Korea
}

Received December 1, 2016; Accepted January 30, 2017

DOI: $10.3892 /$ br.2017.864

\begin{abstract}
Trimethyltin (TMT) is a potent neurotoxicant that affects various regions within the central nervous system, including the neocortex, cerebellum, and hippocampus. In the present study, ginsenoside Rd was investigated as a candidate neuroprotective agent in a primary hippocampal neuron culture and mouse models. TMT induced neurotoxicity in a seven-day primary hippocampal neuron culture in a dose-dependent manner (2.5-10 $\mu \mathrm{M})$. However, pre-treatment with $20 \mu \mathrm{g} / \mathrm{ml}$ ginsenoside $\mathrm{Rd}$ for $24 \mathrm{~h}$ reversed the toxic action. ICR mice were administered a single injection of $2 \mathrm{mg} / \mathrm{kg}$ body weight TMT. Apparent tremor seizure and impaired passive avoidance tests demonstrated significant differences when compared with a saline treated control group. Nissl staining was performed to evaluate the neuronal loss in the hippocampus. In addition, immunostaining of glial fibrillary acidic protein characterized the features of astroglial activation. These results demonstrated that TMT markedly induced Cornu Ammonis 1 subregion neuronal loss and reactive astrocytes in the hippocampus, indicating disrupted hippocampal function. Notably, ginsenoside $\mathrm{Rd}$ attenuated the tremor seizures and cognitive decline in behavioral tests. Additionally, significantly reduced neuronal loss $(\mathrm{P}=0.018)$ and active astroglials $(\mathrm{P}=0.003)$ were observed in the ginsenoside $\mathrm{Rd}$ treated group. Ginsenoside $\mathrm{Rd}$ prevented TMT-induced cell apoptosis via regulation of B-cell lymphoma 2 (Bcl-2), bcl-2-like protein 4 and caspase-3. These
\end{abstract}

Correspondence to: Professor Changkeun Sung, Department of Food Science and Technology, College of Agriculture and Biotechnology, Chungnam National University, Building E8, 99 Daehak-ro, Yuseong-gu, Daejeon 305764, Republic of Korea E-mail: sungchangkeun@126.com

${ }^{*}$ Contributed equally

Key words: ginsenoside Rd, trimethyltin, hippocampus, protection, apoptosis results demonstrate that ginsenoside may be developed as a neuroprotective agent to prevent TMT-induced neurotoxicity.

\section{Introduction}

Evidence has been accumulating over several decades that industrial chemicals cause neurodevelopmental damage (1). Trimethyltin (TMT) is a particularly potent organotin chemical, formerly used for a variety of industrial and agricultural purposes, which has been extensively investigated and used as a model neurotoxin for investigating selective brain dysfunction and delayed neuronal cell death (2-4). Various regions known to be affected by TMT include the hippocampus, preform cortex, amygdaloid nucleus, neocortex, basal ganglia, cerebellum, brain stem, spinal cord, dorsal root ganglia, olfactory cortex, retina and inner ear (5). The implications of the hippocampus in memory processes and neurogenesis are well known and it is likely that the hippocampus would be the first region of the brain to be affected by TMT-induced memory and behavioral changes. The molecular pathogenesis of TMT intoxication is hypothesized to be a complex event. Different theories have been proposed to explain the underlying mechanism of the neurotoxic action of TMT, including glutamate excitotoxicity, intracellular calcium overload and impairment of neurotransmission $(6,7)$, along with oxidative stress $(8,9)$. Although the underlying cellular mechanisms remain unknown, TMT undoubtedly causes profound behavioral changes, and affects learning and memory ability in exposed mammals, as indicated by poor performances in the water maze test, Y-maze test and passive avoidance test.

Ginseng is a deciduous perennial plant that belongs to the Araliaceae family. Panax ginseng, cultivated in China, Korea and the USA (Panax quinquefolium L) represents the most extensively investigated species. The pharmacological and therapeutic effects of ginseng are attributed to ginsenosides, which have been demonstrated to affect the central nervous system (CNS), cardiovascular system, endocrine secretion, immune function and metabolism, as well as possessing anti-stress and -aging properties (10-13). Ginsenosides are classified into three major categories, namely protopanaxatriols (PPTs; ginsenoside Rg1, Re, 
Rg2, Rh1 and Rf), protopanaxadiol (PPD; ginsenoside $\mathrm{Rb} 1, \mathrm{Rb} 2, \mathrm{Rd}, \mathrm{Rg} 3$ and Rh2) and oleanolic acid derivates (ginsenoside Ro). Ginsenoside has become the focus of numerous research groups due to its involvement and protective action in various types of manipulated neuronal damage or dysfunction models in vivo and in vitro (14-19). Certain classical models, such as cerebral ischemia $(14,15)$, chemical-induced excitotoxicity $(16,17)$ and Alzheimer's disease $(18,19)$ have received particular attention. However, these lines of evidence support the interaction between ginsenosides and the CNS, indicating that ginsenosides have more potential for other underlying neuronal diseases. Based upon the above-mentioned evidence of ginsenosides in the CNS, the present study examined the impact of ginsenoside $\mathrm{Rd}$ on TMT-induced neurotoxicity to elucidate the potential neuroprotective potential.

\section{Materials and methods}

Chemicals. TMT chloride was purchased from Sigma-Aldrich (Merck KGaA, Darmstadt, Germany). Ginsenoside Rd, with a purity of $>98 \%$, was generously provided by the Central Research Institute of KT\&G (Daejeon, South Korea). Ginsenoside was dissolved in dimethyl sulfoxide as a $10-\mathrm{mg} / \mathrm{ml}$ solution. All other chemicals in the present study were of analytical grade.

Animals. Male ICR mice (age, 8 weeks; n=35-40) were obtained from Dahan Biolink (Eumseong, South Korea) in present study. The mice were housed in groups in a room maintained at $24 \pm 2^{\circ} \mathrm{C}$ under artificial lighting from 7:00 a.m. to 7:00 p.m., with free access to food and water. All experimental procedures complied with the Guide for the Care and Use of Laboratory Animals issued by the National Institutes of the Committee of Animal Experiments (Chungnam National University, Daejeon, South Korea).

Primary culture of hippocampal neurons. Hippocampi were aseptically dissected from Sprague-Dawley rat embryos (embryonic day 18) acquired from OrientBio, Inc. (Seongnam, South Korea). Hippocampal neurons were dissociated from the tissue samples as previously described (20). Following trituration and trypsinization, hippocampal cells were resuspended in plating medium (86.55\% Minimum Essential Medium Eagle with Earle's Balanced Salt Solution, $10 \%$ re-filtered and heat inactivated fetal bovine serum, $0.45 \%$ glucose, $100 \mu \mathrm{M}$ sodium pyruvate, $200 \mu \mathrm{M}$ glutamine, and $100 \mathrm{mg} / \mathrm{l}$ streptomycin and $100 \mathrm{U} / \mathrm{ml}$ penicillin). The single-cell suspension was seeded in $100-\mathrm{mm}$ petri-dishes containing poly-L-lysine coated coverslips at a density of $5 \times 10^{5} / \mathrm{ml}$ (pre-warmed at $37^{\circ} \mathrm{C}$ ). After $4 \mathrm{~h}$, the cells were maintained in Neurobasal Medium (21103-049; Invitrogen; Thermo Fisher Scientific, Inc, Waltham, MA, USA) supplemented with $1 \% \mathrm{~B} 27,200 \mu \mathrm{M}$ glutamine and $100 \mathrm{mg} / \mathrm{l}$ streptomycin, $100 \mathrm{U} / \mathrm{ml}$ penicillin in a humidified atmosphere of $5 \% \mathrm{CO}_{2}$ at $37^{\circ} \mathrm{C}$. Protocols were performed in accordance with the national guidelines governing animal care in South Korea.

Experimental protocol. TMT chloride (0.1-10 $\mu \mathrm{M})$ was added to the 7-day primary cultures of in vitro hippocampal neurons for $24 \mathrm{~h}$ with or without ginsenoside $\mathrm{Rd}(1-40 \mu \mathrm{g} / \mathrm{ml})$ pre-treatment for $24 \mathrm{~h}$. Then cells were subjected to cell viability assay.

The entire timescale was 3 weeks. Mice received a single injection of TMT chloride ( $2 \mathrm{mg} / \mathrm{kg} /$ body weight) dissolved in sterile saline immediately (intraperitoneally) on day 14 , while the ginsenoside $\mathrm{Rd}$ (20 mg/kg/body weight) was intraperitoneally injected for 21 consecutive days. Control mice received $0.9 \%$ sterile saline injections (intraperitoneally) of the same volume. The treatment groups were as follows: Saline-treated group (Con; $\mathrm{n}=8$ ); TMT chloride-treated animals (TMT; $\mathrm{n}=10$ ); ginsenoside Rd-treated group ( $\mathrm{Rd} ; \mathrm{n}=8)$; and TMT and ginsenoside Rd co-treated group (TMT + Rd; $n=9)$.

Assessment of tremor/seizure severity. Twenty-four hours post-injection, all mice were scored during a 5-min interval on a tremor/seizure severity scale (21) as follows: 1, normal behavior; 2, hyper-responsiveness to sound and handling; 3 , whole body mild tremor with normal motor activity; 4 , whole body tremor with extended periods of immobility; 5 , rigid posture; 6 , forelimb clonus, rearing and falling; 7 , repeated incidence of level 4 behavior; and 8 , severe tonic-clonic behavior.

Passive avoidance test. A passive avoidance test was performed at day 21 in identical compartments. The illuminated compartment $(20 \times 20 \times 20 \mathrm{~cm})$ contained a $100-\mathrm{W}$ bulb, and the floor of the non-illuminated compartment $(20 \times 20 \times 20 \mathrm{~cm})$ was composed of 2-mm stainless steel rods with $1-\mathrm{cm}$ intervals. These two compartments were separated by a guillotine door $(5 \times 5 \mathrm{~cm})$. For the acquisition trials, mice were initially placed in the illuminated compartment and the door was opened $15 \mathrm{sec}$ later. When the mouse entered the non-illuminated compartment, the door was closed and an electrical foot shock $(0.5 \mathrm{~mA})$ of $5 \mathrm{sec}$ duration was delivered through the stainless steel rods. Twenty-four hours after the acquisition trials, the mice were placed in the illuminated compartment again for the retention trials. The time taken for a mouse to enter the non-illuminated compartment after the door was opened was defined as the step-through latency time in the retention trials. If a mouse did not enter the non-illuminated compartment within $300 \mathrm{sec}$, it was assumed that the mouse had remembered the single training trial.

Immunohistochemistry. Coronal sections $(8 \mu \mathrm{m})$ were sliced using a microtome and deparaffinized according to standard protocols. To reveal the morphological changes induced by TMT, Nissl staining of hippocampal sections was performed. The population and morphology of neurons and astrocytes in tissue samples were identified immunocytochemically using anti-glial fibrillary acidic protein (GFAP) antibody (astroglial cells; cat. no. sc-166458; Santa Cruz Biotechnology, Inc., Dallas, TX, USA). Sections were placed in citrate buffer (0.01 M; pH 6.0) and incubated at $80^{\circ} \mathrm{C}$ for $30 \mathrm{~min}$. The sections were washed with phosphate-buffered saline (PBS) twice, for 5 min each time, and blocked with $3 \%$ fetal bovine serum blocking solution for $30 \mathrm{~min}$, which was followed by incubation with primary anti-body GFAP (dilution, 1:50) overnight at $4^{\circ} \mathrm{C}$. The sections were counterstained with hematoxylin and cover slipped using histomount mounting solution. 
Image acquisition and analysis. A Zeiss Research Microscope was used for phase-contrast microscopy. Morphometric analyses and quantification were performed using Image J software (version 1.50i) with the simple neurite tracer plug-in (National Institute of Health, Bethesda, MD, USA). The lengths of primary dendrites and axons were analyzed.

The sections were analyzed using an Olympus microscope equipped with a digital camera (Olympus Corporation, Tokyo, Japan). The cells were counted by a researcher who was blinded to the experimental conditions. Every ten sections throughout the hippocampus were processed for counting (5-6 sections per animal).

Western blotting. Hippocampi were dissected and homogenized in protein extraction buffer (PRO-PREP ${ }^{\text {Tм }} 17081$; iNtRON Biotechnology, Seongnam, South Korea). The supernatant was collected following centrifugation at 9,740 $\mathrm{x} g$ for $30 \mathrm{~min}$ at $4^{\circ} \mathrm{C}$. Following quantification, the samples $(20 \mu \mathrm{g}$ protein per lane) were subjected to preparative sodium dodecyl sulfate-polyacrylamide gel electrophoresis in a $10 \%$ gel and electrophoretically transferred onto polyvinylidene fluoride (PVDF) membranes (EMD Millipore, Billerica, MA, USA) using a trans-blot device (Bio-Rad Laboratories, Inc., Hercules, CA, USA) at a constant current of $15 \mathrm{~V}$ overnight at $4^{\circ} \mathrm{C}$. The PVDF membranes were soaked in $5 \%$ skimmed milk in PBS solution for $2 \mathrm{~h}$ at room temperature to block non-specific binding, rinsed in PBS with Tween-20 (PBST), and incubated with B-cell lymphoma 2 (Bcl-2; cat. no. 2876), bcl-2-like protein 4 (Bax; cat. no. 5023S) and caspase-3 (cat. no. 9664S) antibodies (diluted 1:1,000 in 5\% skimmed milk in TBST; Cell Signaling Technology, Inc., Danvers, MA, USA) overnight at $4^{\circ} \mathrm{C}$. The membranes were then washed three times (10 min each time) in PBST and incubated for $2 \mathrm{~h}$ with goat anti-rabbit immunoglobulin $\mathrm{G}$ secondary antibody (dilution, 1:5,000; cat. no. sc-2030; Santa Cruz Biotechnology, Inc.). Western blot analysis for GAPDH, the loading control (dilution, 1:4,000; cat. no. sc-25778; Santa Cruz Biotechnology, Inc.), was performed using the same procedure. The blots were quantified using ImageJ image analysis software (version 1.50i). Band intensity values were expressed as a percentage of the control average.

Statistical analysis. All statistical analyses were conducted using SPPSS version 20 (IBM SPSS, Armonk, NY, USA). $\mathrm{P} \leq 0.05$ was considered to indicate a statistically significant difference. When a statistically significant overall group effect was found, multiple comparisons were made using Fisher protected least significant different post hoc tests to compare the individual groups.

\section{Results}

TMT induces apoptosis of primary hippocampal neurons in culture. No morphological changes were observed in the neurons following exposure to $0-1 \mu \mathrm{M}$ TMT for $24 \mathrm{~h}$, as revealed by phase contrast microscopy. Application of greater TMT concentrations $(0,2.5,5.0$ and $10.0 \mu \mathrm{M})$ induced a dose-dependent increase of neuronal degeneration manifested by neurite fragmentation and regression, as well as somal rounding and shrinkage (data not shown). Dose-dependent
A

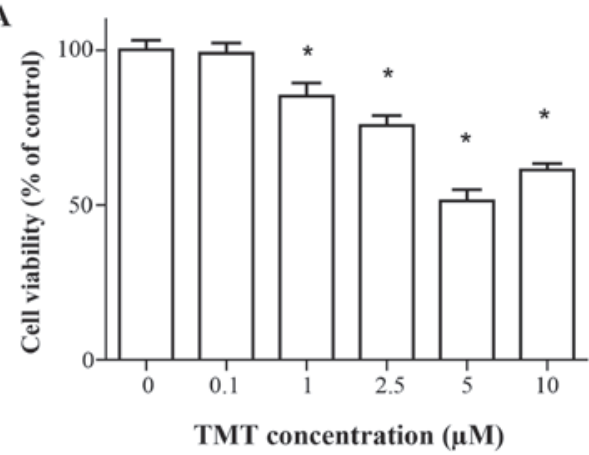

B

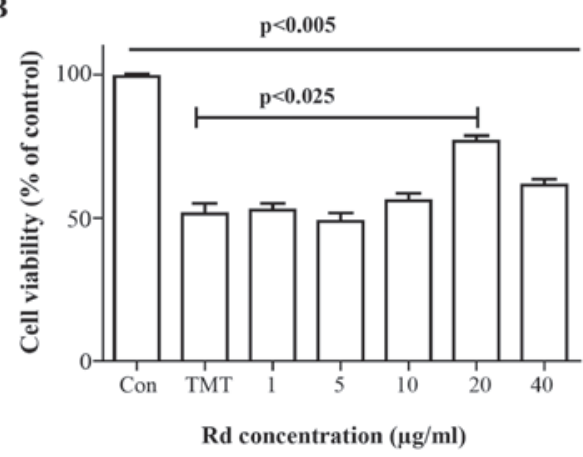

Figure 1. TMT induced apoptosis in primary hippocampal neurons and this neurotoxicity was reversed by pre-treatment with ginsenoside $\mathrm{Rd}$ for $24 \mathrm{~h}$. The dose-dependent effect on neuronal cell viability of (A) TMT and (B) ginsenoside Rd pre-treatment followed by $5 \mu \mathrm{M}$ TMT induction. Each bar represents the mean \pm standard error of the mean. ${ }^{*} \mathrm{P}<0.05$ vs. $0 \mu \mathrm{M}$ (Con). TMT, trimethyltin; Con, control; Rd, ginsenoside Rd.

effects of TMT on neuronal viability are presented in Fig. 1A. To further analyze the protective effects of Rd on TMT-induced cytotoxicity, the percentage of apoptotic cells with obvious morphological changes following exposure to $5 \mu \mathrm{M}$ TMT for $24 \mathrm{~h}$ was quantified. To optimize the effective concentration, hippocampal neurons were treated with different concentrations of ginsenoside Rd. Ginsenoside Rd treatment (in the range of $10-40 \mu \mathrm{M}$ ) significantly increased the cell viability (including neurite outgrowth) in a dose-dependent manner, whereas no significant effects were noted following treatment with 1 or $5 \mu \mathrm{M}$ ginsenoside Rd. An optimal concentration of $20 \mu \mathrm{g} / \mathrm{ml}$ pre-treatment for $24 \mathrm{~h}$ was proposed herein (Fig. 1B). Together, these data indicate that apoptotic cell death induced by TMT was efficiently prevented by ginsenoside Rd pre-treatment.

TMT-induced behavior changes. Within $24 \mathrm{~h}$ of TMT exposure, mice exhibited clinical signs of tremor and seizure activity that were attenuated by ginsenoside Rd treatment (Fig. 2A). The seizure score of the TMT group was significantly greater $(\mathrm{P}<0.0001)$ than that of the control. The seizure score was significantly reduced in the TMT+Rd mice $(\mathrm{P}<0.0001)$ when compared with the TMT mice. Furthermore, the Rd alone group did not differ from the control group.

Significant overall group effects were observed in the passive avoidance test $(\mathrm{P}=0.025)$ subsequent to a three-week treatment. In the passive avoidance test (Fig. 2B), although TMT-treated mice exhibited significantly decreased step-through latency $(\mathrm{P}=0.009)$ compared with the control group mice, no significant differences were noted in the TMT+Rd $(20 \mathrm{mg} / \mathrm{kg} ; \mathrm{P}=0.325)$ and $\mathrm{Rd}(\mathrm{P}=0.124)$ groups when compared with the control group. 
A

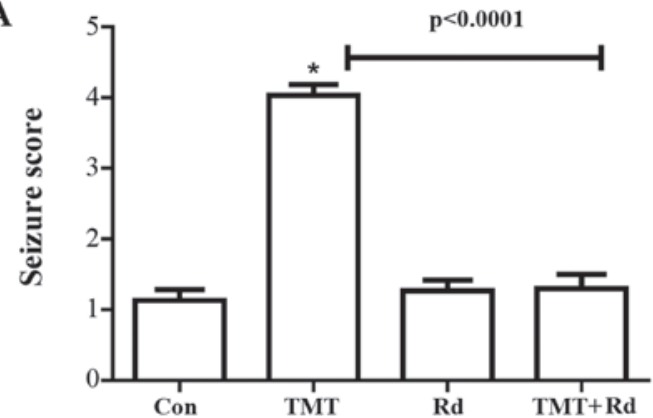

B

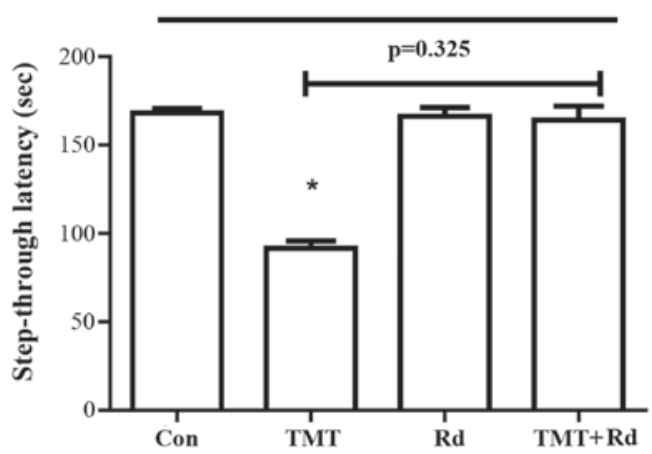

Figure 2. (A) TMT induced tremors and seizures in mice at $24 \mathrm{~h}$ post-injection. (B) Effects of ginsenoside Rd on spatial memory of mice exposed to TMT. ${ }^{*} \mathrm{P}<0.05$ vs. Con. TMT, trimethyltin; Con, saline control; Rd, ginsenoside Rd.

TMT induces neuronal loss in the hippocampus and astroglial activation. To investigate the contribution of ginsenoside $\mathrm{Rd}$ on the protection of hippocampal neurons, mice were treated with ginsenoside $\mathrm{Rd}$ (20 mg/kg body weight) for 21 days. As revealed by Nissl staining (Fig. 3A-D), treatment with TMT induced the loss of hippocampal Cornu Ammonis 1 (CA1) subregion cells, although this was not observed in the Rd+TMT group. As demonstrated in Fig. 3E, the number of pyramidal neurons in the CA1 subregions was determined. The neuronal loss indicated the marked toxicity of TMT in the hippocampus.

GFAP immunocytochemical staining demonstrated that TMT induced a marked increase of GFAP immunoreactivity in the entire hippocampus, with the most significant expression observed in the astrocytes of the dentate gyrus (Fig. 3F-I). The morphology of astrocytes and intensive astrofilament staining indicated hypertrophic activity of astroglia. As can be seen in Fig. 3J, the TMT group had significantly more $\mathrm{GFAP}^{+}$cells than the saline-treated control group $(\mathrm{P}<0.004)$. In contrast to this observation, the $\mathrm{Rd}$ and $\mathrm{TMT}+\mathrm{Rd}$ groups were not significantly different from the controls $(\mathrm{P}=0.34)$.

Ginsenoside Rd alters apoptosis induced by TMT in the hippocampus. Induction of apoptosis, the cellular waste disposal process, was evaluated by the protein expression of $\mathrm{Bcl}-2$, Bax, and caspases-3. The expression level of Bcl-2 was identified to be significantly reduced by TMT when compared with that of the control group $(\mathrm{P}<0.05)$. By contrast, the expression levels of Bax and caspase- 3 were significantly increased in the TMT group $(\mathrm{P}<0.05)$, indicating that apoptosis was induced in the hippocampus. Notably, treatment with ginsenoside

Rd reversed the decrease of Bcl-2 and increase of Bax and caspase-3 expression levels, indicating a neuronal protective effect (Fig. 4).

\section{Discussion}

The current study demonstrated that exposure to TMT alters the physical condition (tremor/seizure test) and spatial recognition memory (passive avoidance test), which are associated with hippocampal dysfunction. Consistent with functional deficits, significant neuron loss and astroglial activation were observed in the hippocampus of treated mice. In addition, this cytotoxicity was confirmed in the primary hippocampal neuron culture system. Notably, there was a pronounced protective effect of ginsenoside $\mathrm{Rd}$ in the TMT exposure models. Pre-treatment with $20 \mu \mathrm{g} / \mathrm{ml}$ ginsenoside $\mathrm{Rd}$ for $24 \mathrm{~h}$ markedly reduced cell death $(\sim 25 \%)$ compared with TMT treatment alone, indicating that ginsenoside $\mathrm{Rd}$ protected against TMT-induced apoptosis.

Animals exposed to TMT suffer spontaneous seizures and elevation of neuronal excitability $(22,23)$. In the present study, whole body tremor, with extended periods of immobility, was observed in mice that received TMT injections. Additionally, the toxic interaction of TMT with the hippocampus and other limbic brain regions is responsible for affecting spatial learning and memory. Deficits in passive avoidance behavior result from TMT exposure $(24,25)$, which is consistent with the observations in the current study. To explain any potential protection against these functional decrements, the current study hypothesized that a specific protection of ginsenoside $\mathrm{Rd}$ would adversely impact hippocampal impairment. Significant reduction in tremor seizures, and improved learning and memory ability suggest that ginsenoside $\mathrm{Rd}$ attenuates toxicity herein.

The impact of TMT on neurons was assessed in the current study using a primary hippocampal neuron culture and mouse models. In the present study, TMT induced primary hippocampal neuron cell death in a dose-dependent manner. In the animal model, TMT treatment developed extensive lesions in the CA. Furthermore, apparent neuronal loss was observed in the CA1 subregion in the hippocampus, in the present study, which was confirmed by Nissl staining. The present results are consistent with those of previous studies $(5,26)$, indicating that hippocampal vulnerability is typical in TMT exposure. Furthermore, TMT-induced neuronal loss is associated with the activation of astrocytes and GFAP expression levels are altered in the hippocampus following TMT treatment. Various studies have established the active role of astrocytes in TMT-induced neurodegeneration. Certain studies indicated that reactive astrocytes express a trophic response, such as nerve growth factor and tropomyosin receptor kinase A to TMT exposure (27). Certain studies proposed an involvement of $\mathrm{P} 2 \mathrm{X} 2$ purinergic receptor signaling induction from astroglial (28). In addition, the role of astroglia in preventing TMT-induced neuronal cell death by modulating oxidative stress was also proposed (29). Notably, the present data identified similar GFAP expression levels between the control group and the TMT+Rd group. Thus, it may indicate that ginsenoside Rd prevented the TMT-induced neuronal toxicity, rather than detoxication. Previous findings suggest a direct involvement of mitochondrial function in 
A

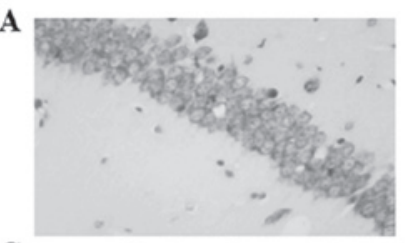

C

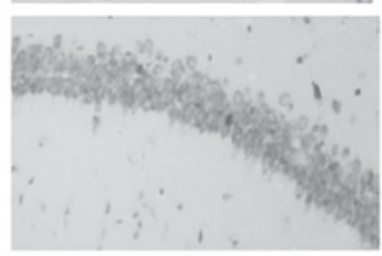

F

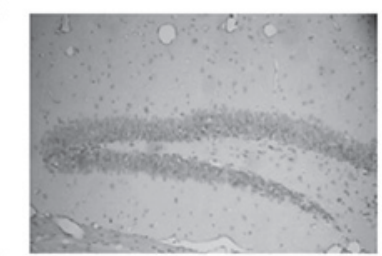

H

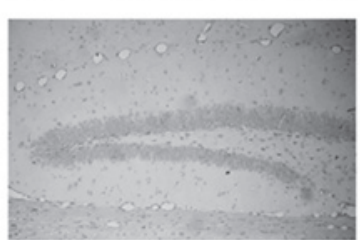

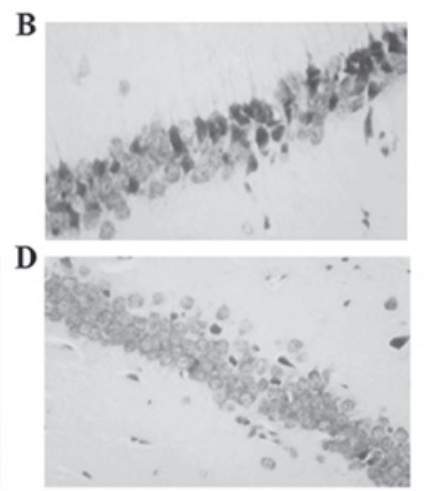

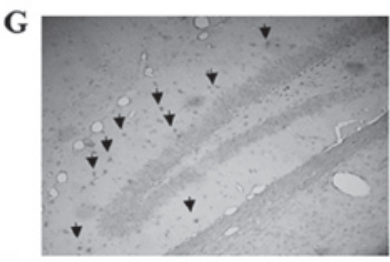

I

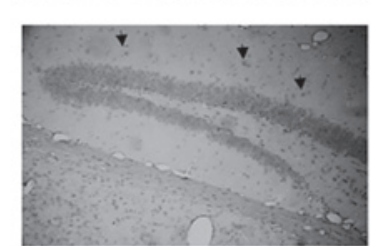

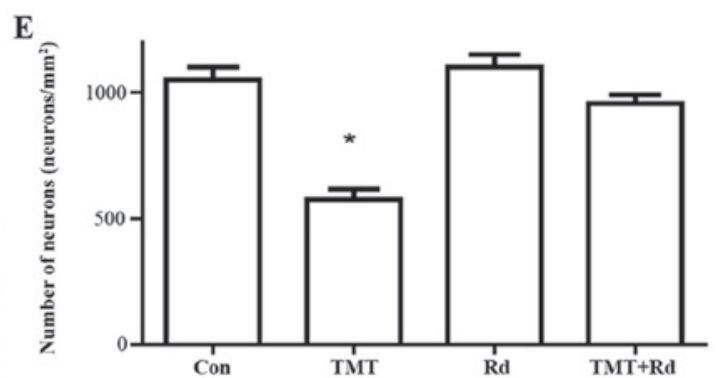

$\mathbf{J}$

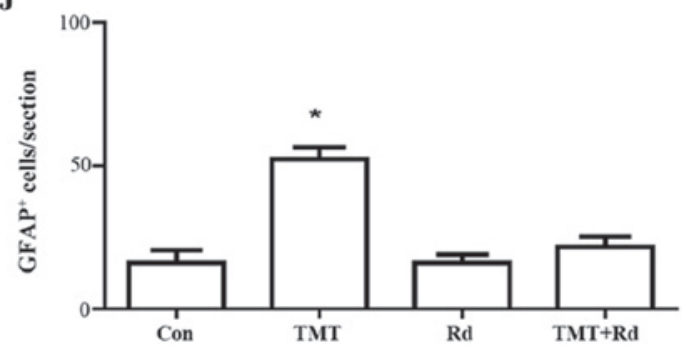

Figure 3. Treatment with TMT induced hippocampal subregion CA1 neuron loss and astrocyte activation. Nissl staining for coronal sections from the (A) control, (B) TMT, (C) Rd and (D) TMT + Rd groups. Original magnification, x100. (E) The number of pyramidal neurons in the CA1 subregion of the hippocampus was determined. Each bar represents the mean \pm standard error of the mean. Glial fibrillary acidic protein immunostaining for coronal sections from the (F) control, (G) TMT, (H) Rd and (I) TMT + Rd groups. The arrows indicate positive cells. Original magnification, x60. (J) Quantification of GFAP. Each bar represents the mean \pm standard error of the mean. ${ }^{*}<0.05$ vs. Con. TMT, trimethyltin; CA1, Cornu Ammonis 1; Con, control; Rd, ginsenoside Rd.

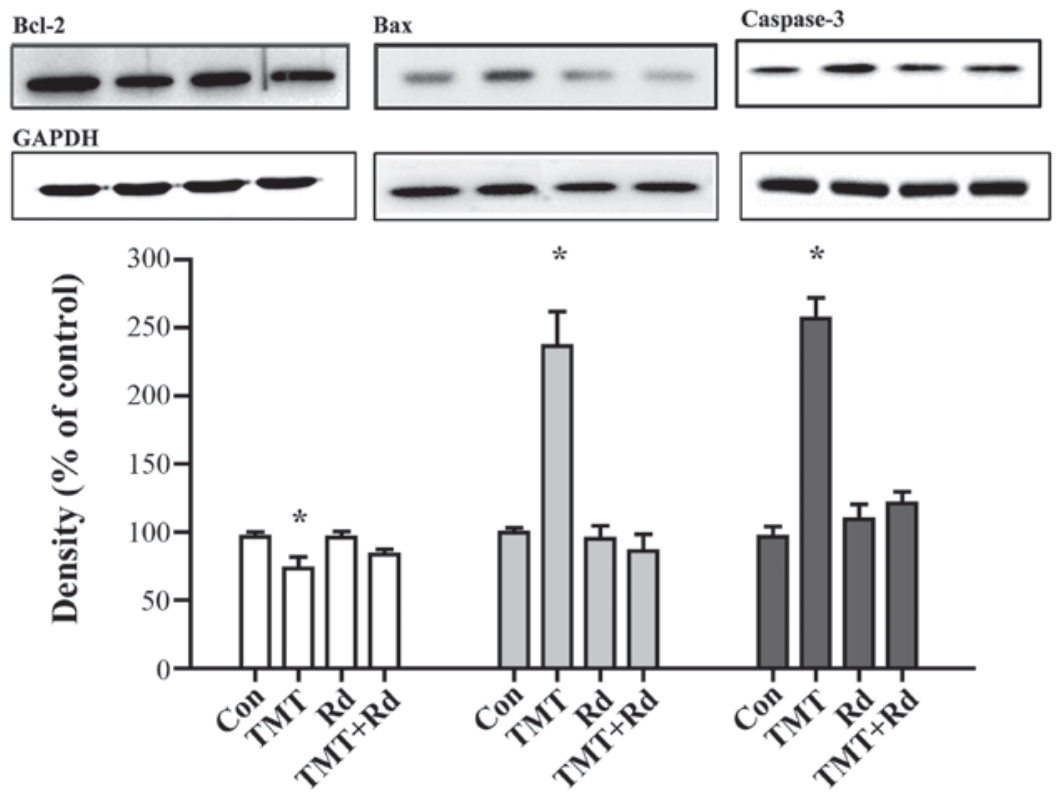

Figure 4. Effects of ginsenoside Rd on expression levels of Bcl-2, Bax and caspase-3 in the hippocampus of mice subjected to TMT exposure. Each bar represents the mean \pm standard error of the mean. ${ }^{*} \mathrm{P}<0.05$ vs. Con. Bcl-2, B-cell lymphoma 2; Bax, Bcl-2-like protein 4; TMT, trimethyltin; Con, control; Rd, ginsenoside Rd.

response to TMT $(30,31)$. The present study hypothesized that the effectors, Bcl-2 and Bax, in the intrinsic (mitochondrial mediated) apoptotic pathway were modulated by ginsenoside Rd treatment, indicating a potential molecular mechanism.
In conclusion, the present study demonstrated the deleterious effect of TMT on neurons, which resulted in hippocampal dysfunction. Treatment with ginsenoside Rd may prevent the toxicity in in vitro and in vivo models. These results therefore 
indicate that ginsenoside Rd may be administered as a potential neuroprotective agent.

\section{Acknowledgements}

The present study was supported by the Korean Society of Ginseng and Korean Ginseng Corporation (2013).

\section{References}

1. Dorman DC: An integrative approach to neurotoxicology. Toxicol Pathol 28: 37-42, 2000.

2. Ha JS, Jin DE, Park SK, Park CH, Seung TW, Bae DW, Kim DO and Heo HJ: Antiamnesic Effect of Actinidia arguta Extract Intake in a Mouse Model of TMT-Induced Learning and Memory Dysfunction. Evid Based Complement Alternat Med 2015 876484, 2015.

3. Lattanzi W, Corvino V, Di Maria V, Michetti F and Geloso MC: Gene expression profiling as a tool to investigate the molecular machinery activated during hippocampal neurodegeneration induced by trimethyltin (TMT) administration. Int J Mol Sci 14: 16817-16835, 2013.

4. Kim J, Yang M, Kim J, Song L, Lee S, Son Y, Kang S, Bae CS, Kim JC, Kim SH, et al: Developmental and degenerative modulation of brain-derived neurotrophic factor transcript variants in the mouse hippocampus. Int J Dev Neurosci 38: 68-73, 2014.

5. Bouldin TW, Goines ND, Bagnell RC and Krigman MR: Pathogenesis of trimethyltin neuronal toxicity. Ultrastructural and cytochemical observations. Am J Pathol 104: 237-249, 1981.

6. Park HJ, Lee MS, Shim HS, Lee GR, Chung SY, Kang YM, Lee BJ, Seo YB, Kim KS and Shim I: Fermented Saccharina japonica (Phaeophyta) improves neuritogenic activity and TMT-induced cognitive deficits in rats. Algae 31: 73-84, 2016.

7. Shim HS, Park HJ, Ahn YH, Her S, Han JJ, Hahm DH, Lee H and Shim I: Krill-Derived Phosphatidylserine Improves TMT-Induced Memory Impairment in the Rat. Biomol Ther (Seoul) 20: 207-213, 2012.

8. Kaur S and Nehru B: Alteration in glutathione homeostasis and oxidative stress during the sequelae of trimethyltin syndrome in rat brain. Biol Trace Elem Res 153: 299-308, 2013.

9. Gasparova Z, Stara V, Janega P, Navarova J, Sedlackova N, Mach M and Ujhazy E: Pyridoindole antioxidant-induced preservation of rat hippocampal pyramidal cell number linked with reduction of oxidative stress yet without influence on cognitive deterioration in Alzheimer-like neurodegeneration. Neuro Endocrinol Lett 35: 454-462, 2014.

10. Kim HJ, Kim P and Shin CY: A comprehensive review of the therapeutic and pharmacological effects of ginseng and ginsenosides in central nervous system. J Ginseng Res 37: 8-29, 2013.

11. Peng L, Sun S, Xie LH, Wicks SM and Xie JT: Ginsenoside Re: Pharmacological effects on cardiovascular system. Cardiovasc Ther 30: e183-e188, 2012.

12. Kim MY and Cho JY: 20S-dihydroprotopanaxadiol, a ginsenoside derivative, boosts innate immune responses of monocytes and macrophages. J Ginseng Res 37: 293-299, 2013.

13. Wang Y, Kan H, Yin Y, Wu W, Hu W, Wang M, Li W and Li W: Protective effects of ginsenoside Rg1 on chronic restraint stress induced learning and memory impairments in male mice. Pharmacol Biochem Behav 120: 73-81, 2014.

14. Zhou Y, Li HQ, Lu L, Fu DL, Liu AJ, Li JH and Zheng GQ: Ginsenoside Rg1 provides neuroprotection against blood brain barrier disruption and neurological injury in a rat model of cerebral ischemia/reperfusion through downregulation of aquaporin 4 expression. Phytomedicine 21: 998-1003, 2014.
15. Wang Y, Li X, Wang X, Lau W, Wang Y, Xing Y, Zhang X, Ma X and Gao F: Ginsenoside Rd attenuates myocardial ischemia/reperfusion injury via Akt/GSK-3 $\beta$ signaling and inhibition of the mitochondria-dependent apoptotic pathway. PLoS One 8: e70956, 2013.

16. Zhang C, Du F, Shi M, Ye R, Cheng H, Han J, Ma L, Cao R, Rao $\mathrm{Z}$ and Zhao G: Ginsenoside Rd protects neurons against glutamate-induced excitotoxicity by inhibiting ca(2+) influx. Cell Mol Neurobiol 32: 121-128, 2012.

17. Li G, Zhang XX, Lin L, et al: Preparation of Ginsenoside Rg3 and Protection against $\mathrm{H}_{2} \mathrm{O}_{2}$-Induced Oxidative Stress in Human Neuroblastoma SK-N-SH Cells. J Chem 2014: 848571, 2014.

18. Li N, Zhou L, Li W, Liu Y, Wang J and He P: Protective effects of ginsenosides Rg1 and Rbl on an Alzheimer's disease mouse model: A metabolomics study. J Chromatogr B Analyt Technol Biomed Life Sci 985: 54-61, 2015.

19. Fang F, Chen X, Huang T, Lue LF, Luddy JS and Yan SS: Multi-faced neuroprotective effects of Ginsenoside Rg1 in an Alzheimer mouse model. Biochim Biophys Acta 1822: 286-292, 2012.

20. Kaech S and Banker G: Culturing hippocampal neurons. Nat Protoc 1: 2406-2415, 2006.

21. Funk JA, Gohlke J, Kraft AD, McPherson CA, Collins JB and Jean Harry G: Voluntary exercise protects hippocampal neurons from trimethyltin injury: Possible role of interleukin-6 to modulate tumor necrosis factor receptor-mediated neurotoxicity. Brain Behav Immun 25: 1063-1077, 2011.

22. Ishida N, Akaike M, Tsutsumi S, Kanai H, Masui A, Sadamatsu M, Kuroda Y, Watanabe Y, McEwen BS and Kato N: Trimethyltin syndrome as a hippocampal degeneration model: Temporal changes and neurochemical features of seizure susceptibility and learning impairment. Neuroscience 81: 1183-1191, 1997.

23. Janigro D and Costa LG: Effects of trimethyltin on granule cells excitability in the in vitro rat dentate gyrus. Neurotoxicol Teratol 9: 33-38, 1987.

24. Choi GN, Kim JH, Kwak JH, Jeong CH, Jeong HR, Lee U and Heo HJ: Effect of quercetin on learning and memory performance in ICR mice under neurotoxic trimethyltin exposure. Food Chem 132: 1019-1024, 2012.

25. Kaur S, Chhabra R and Nehru B: Ginkgo biloba extract attenuates hippocampal neuronal loss and cognitive dysfunction resulting from trimethyltin in mice. Phytomedicine 20: 178-186, 2013.

26. Geloso MC, Vercelli A, Corvino V, Repici M, Boca M, Haglid K, Zelano $G$ and Michetti F: Cyclooxygenase-2 and caspase 3 expression in trimethyltin-induced apoptosis in the mouse hippocampus. Exp Neurol 175: 152-160, 2002.

27. Koczyk D and Oderfeld-Nowak B: Long-term microglial and astroglial activation in the hippocampus of trimethyltin-intoxicated rat: Stimulation of NGF and TrkA immunoreactivities in astroglia but not in microglia. Int J Dev Neurosci 18: 591-606, 2000.

28. Latini L, Geloso MC, Corvino V, Giannetti S, Florenzano F, Viscomi MT, Michetti F and Molinari M: Trimethyltin intoxication up-regulates nitric oxide synthase in neurons and purinergic ionotropic receptor 2 in astrocytes in the hippocampus. J Neurosci Res 88: 500-509, 2010.

29. Gunasekar PG, Mickova V, Kotyzova D, Li L, Borowitz JL, Eybl V and Isom GE: Role of astrocytes in trimethyltin neurotoxicity. J Biochem Mol Toxicol 15: 256-262, 2001.

30. Qu M, Zhou Z, Chen C, Li M, Pei L, Chu F, Yang J, Wang Y, Li L, Liu C, et al: Lycopene protects against trimethyltin-induced neurotoxicity in primary cultured rat hippocampal neurons by inhibiting the mitochondrial apoptotic pathway. Neurochem Int 59: 1095-1103, 2011.

31. Zhang L, Li L, Prabhakaran K, Borowitz JL and Isom GE: Trimethyltin-induced apoptosis is associated with upregulation of inducible nitric oxide synthase and Bax in a hippocampal cell line. Toxicol Appl Pharmacol 216: 34-43, 2006. 